\title{
Ablative therapy in 2016 for small renal masses
}

\begin{abstract}
Introduction: Incidental diagnosis of small renal masses (SRMs), less than $4 \mathrm{~cm}$ in size, is increasing due to the widespread use of different forms of diagnostic imaging. The natural history of these tumours remains unknown and there is no reliable way to predict their behaviour or growth. Partial nephrectomy is currently the gold standard in the treatment of these tumours. However, the more minimally invasive, non- excisional, ablative therapies such as cryoablation (CRA) and radiofrequency ablation (RFA) are providing comparable oncological outcomes.
\end{abstract}

Methods: This article critically reviews the principles, indications, modalities of treatment, oncological outcome, complication and effect on renal function, of the different ablative therapies.

Results: Cryotherapy induces tissue ablation by freezing and thawing using argon and helium gases respectively.RFA ablates the tissue by heating. These Ablative treatments for SRMs are particularly indicated in the elderly patients, those with co-morbidities, in patients with solitary kidneys and in those with impaired renal function. The procedure related complication rate with ablative therapies are lower. These procedures have a promising medium term oncological outcome, while longer- term results are awaited. It appears that Cryotherapy may be evolving as a better modality for oncological control than RFA.

Conclusion: The Ablative therapies for SRMs are emerging as viable treatment options with recurrence free survival rates approaching that of extirpative surgery. However, there is currently no consensus in the literature on the best selection criteria for this form of treatment. With further refinement of selection criteria and more prospective long- term data on the oncological outcome, these ablative methods would have a promising future in the management of SRMs.

Keywords: Ablative therapy; Cryoablation; Cryotherapy; Radiofrequency ablation; Nephron sparing surgery
Volume 4 Issue I - 2017

\section{Pandian S, Farrag K, Sriprasad S}

Department of Urology and Nephrology, Darent Valley Hospital, UK

Correspondence: S Sriprasad, Department of Urology and Nephrology, Darent Valley Hospital, Darenth Wood Road, Dartford, Kent, UK, Tel 00 44I322428780, Fax 00 447774232125, Email sspandian@aol.com

Received: March II, 2016 | Published: January 12, 2017
Abbreviations: SRMs, small renal masses; CRA, cryoablation; RFA, radiofrequency ablation; PN, partial nephrectomy; LPN, laparoscopic partial nephrectomy; RAPN, robotic assisted partial nephrectomy; RFA, radio frequency ablation; HIFU, high intensity focused ultrasound; CT, computerized tomography; MRI, magnetic resonance imaging; US, ultrasound; RVS, real-time virtual ultrasonography; LCA, laparoscopic cryoablation; DFS, disease-free survival; OS, overall survival; IQR, interquartile; RCC, renal cell carcinoma; ASA, American society of anesthesiologists

\section{Introduction}

The wide spread use of advanced diagnostic imaging, has lead to an increase in identifying localized small renal masses (SRM) of less than $4 \mathrm{~cm}$ size (stage T1). ${ }^{1}$ The natural history of these incidentally diagnosed SRMs is not clear. ${ }^{2}$ According to a meta-analysis by Chawla et al. ${ }^{3}$ the median rate of growth for SRMs is $0.28 \mathrm{~cm} /$ year, based on radiological evidence. The gold standard for treatment of SRMsis currently Partial nephrectomy (PN), with oncological outcomes comparable to radical nephrectomy, but with better preservation of the renal function. ${ }^{4-7} \mathrm{PN}$ can be accomplished by either open, laparoscopic or robot assisted methods. Laparoscopic partial nephrectomy (LPN) is technically more demanding with a steep learning curve and has a significant complication rate. ${ }^{7}$ Robotic assisted partial nephrectomy (RAPN) has made this procedure much easier. ${ }^{8}$
However, RAPN still has a learning curve and warm ischemia time to contend with, making it difficult, especially in patients with severe comorbidity. ${ }^{9}$ Ablative therapy, on the other hand, provides a less invasive treatment option in this group of patients with reasonable oncological control comparable to partial nephrectomy. ${ }^{10}$ Cryoablation and Radiofrequency Ablation (RFA) are the two most popular ablative therapy techniques, currently. Other new techniques in the horizon include High Intensity Focused Ultrasound (HIFU), Interstitial Laser Ablation Therapy and Microwave Thermotherapy. However, these new technologies lack clear evidence currently and can largely be considered as experimental and evolving. ${ }^{11}$ This review mainly examines the role of cryotherapy and RFA in the management of SRMs.

\section{Cryotherapy}

The principle of tissue destruction by freezing and thawing using vacuum -insulated liquid argon gas system is used in Cryotherapy. Rapid cooling of the tip of the cryoprobe is achieved using compressed argon gas expanding from its liquid form through the narrow orifice of the probe to a gaseous state. This can achieve a very low temperature on a certain focus in the kidney tissue to freeze the tumour. ${ }^{12}$ The thawing phase follows the cooling phase where helium gas is passed through the probe in the same way to induce thawing. ${ }^{13}$ Acute and delayed tissue damage is induced at the cellular level, by cryoablation. At $-50 \mathrm{C}$, the acute effect takes place, as ice forms in the extracellular 
space changing the osmotic gradient and drawing water from inside the cells to the extracellular space, leading to changes in the intracellular $\mathrm{pH}$ and composition, resulting in protein denaturation. ${ }^{14,15}$ When the temperature reaches $-20^{\circ} \mathrm{C}$, ice forms in both the intra and extracellular spaces, causing irreversible damage to the cell membranes. However, the delayed effect resulting from damage to the microvasculature of the renal parenchyma is believed to be the most important mechanism of action of cryoablation [16]. Majority of times, the repeated cycles of freezing and thawing enhances tissue destruction, with some animal model experiments showing evidence that double freezing lead to large areas of tissue destruction compared to single freezing. ${ }^{17}$ The typical temperature achieved is between $-20^{\circ} \mathrm{C}$ and $-40^{\circ} \mathrm{C}$ or lower, during treatment of SRMs with Cryoablation.

\section{Indications for cryotherapy}

The tumour characteristics suitable for cryotherapy and partial nephrectomy are more or less similar. The typical tumour for cryotherapy is situated in the periphery of the kidney, small $(<4 \mathrm{~cm})$, exophytic, solid and well circumscribed. However, cryoablation is particularly useful in elderly patients or those who have significant comorbidity. The technique may also be considered in small lesions of a solitary or transplant kidney and in conditions with multiple tumours such as tuberous sclerosis or Von Hipple-Lindau disease. ${ }^{18}$ Some centres are routinely considering cryotherapy as another standard treatment option for SRMs and discussing this with the patients. With experience and the availability of laparoscopic ultrasound probe, cystic and solid lesions such as Bosniak 4 type tumours are also treated. We advocate that all cases of SRMs are discussed and evaluated in the cancer multidisciplinary setting and then the most appropriate treatment is recommended to an individual patient. ${ }^{19}$

\section{Techniques of cryotherapy}

Cryotherapy can be performed either by surgical (laparoscopic) approach or using percutaneous radiological techniques. In either approach, the process of delivery of the gases and the control mechanisms are all computerised.

\section{Surgical approach}

Trans or retroperitoneal laparoscopic approach is the common surgical technique employed for cryotherapy. The transperitoneal technique is best suited for anterior tumours. Hilar lesions, which are a relative contraindication foropen/laparoscopic partial nephrectomy, may be suitable for cryoablation. The patient is placed in the lateral position, usually under general anaesthesia, followed by insertion of laparoscopic ports and creation of pnuemoperitoneum. As in laparoscopic nephrectomy, the large bowel is first mobilized medially to expose the Gerota's fascia, which is then opened to reveal the tumour. The exact position and dimensions of the tumour within the kidney is confirmed using intra-operative imaging obtained through a laparoscopic ultrasound probe. Biopsies from the tumour are taken, before the cryotherapy needles (probes) are placed into the tumour. The number of needles required and the depth of their placement are dependent on the size of the tumour. Probes to measure the freezing temperature achieved are also placed, one within the centre of the tumour and the other about a centimetre beyond the tumour circumference. Usually two cycles of freezing with Argon and two cycles of thawing with helium gas are employed. When the tumour is frozen, a spectacular white mound is usually seen at laparoscopy (Figure 1). The needles are removed, post-procedure and any bleeding is usually effectively controlled by compression. The calibre of the needles used and the techniques employed, vary slightly between different machines used. Open surgical cryoablation when necessary, is also another available option.

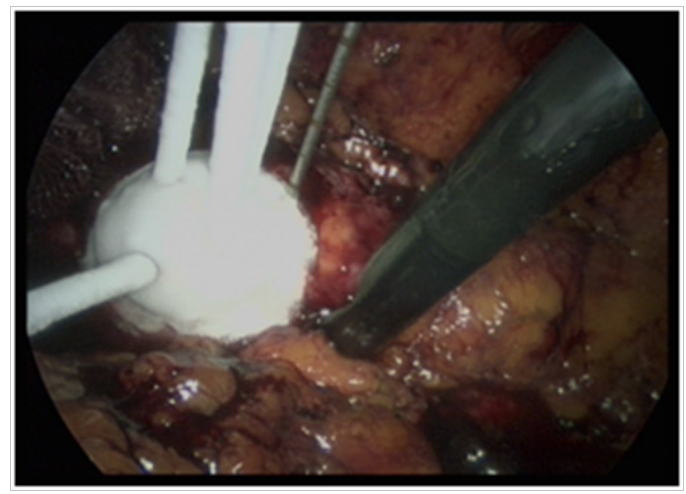

Figure I Laparoscopic cryoprobes placed in renal tumour, producing a white mound.

\section{Percutaneous Approach}

The numbers of patients treated with percutaneous techniques are increasing as the techniques are getting refined. ${ }^{20}$ This approach is best suited for tumours located in the posterior or inferior aspect of the kidney. General anaesthesia is still employed in many centres, as it is uncomfortable for patients to lie still for long periods of time during the treatment. The technique of saline dissection to displace the bowel lying adjacent to kidney, used by expert interventional radiologists, has enabled safe and effective treatment of anterior and superior pole renal tumours (Figure 2). The technique is most commonly performed using Computerized Tomography (CT) scan guidance and less commonly using Magnetic Resonance Imaging (MRI) or ultrasound (US) guidance. The percutaneous approach is associated with lesser hospital stay than laparoscopy and is much safer for patients with significant comorbidity. New imaging modalities are emerging to help improve probe placement and real time intraoperative monitoring. For example, Real-time Virtual Ultrasonography (RVS) is a new modality that utilizes pre-operative CT scan data concomitantly with real time intraoperative ultrasonography. It is a promising tool, to mitigate the deficiencies of conventional ultrasound imaging, including limited visibility and inability of the ultrasound waves to travel through the ice produced bycryoablation. ${ }^{21}$

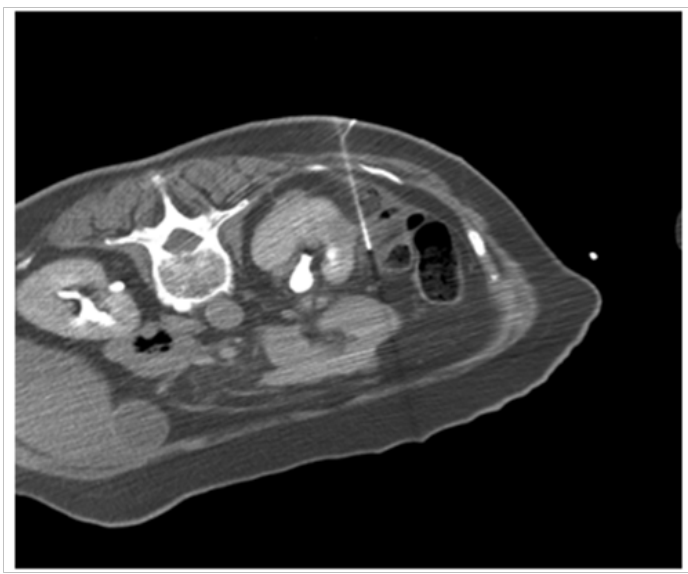

Figure 2 CT-guided cryoablation of a small renal mass using the saline dissection method. 


\section{Complications of Cryoablation}

Cryoablation is minimally invasive and is associated with less number of complications compared to open /laparoscopic / robotic partial nephrectomy and the majority of these are minor complications. Of the 148 cryoablations performed in 144 patients, in a multi-centre study by Laguna et al., ${ }^{22}$ the majority of their reported perioperative complications were classified as Claviengrade I or II. The complications include, minor renal lacerations and bleeding from the needle placement site. The renal fracture risk could be minimized using perpendicular placement of the needles and avoiding removal of these needles before thawing is complete. The probe insertion site at skin level should be protected with gauze or insulating gel placed around the probe. The most common minor complication was pain and paraesthesia at the site of the probe insertion, in another study ${ }^{23}$ of 139 patients. The major complication rate was $1.8 \%$ and included haemorrhage, ileus, conversion to open surgery, scarring with pelviureteric junction obstruction and urinary leakage.

The European Renal Cryoablation Group (EuRECA) recently published its retrospective review of 808 patients. The postoperative complications based on Clavien grade included 74 (11.3\%) patients with Grade I-II and $20(3.1 \%)$ patients with Grade III-V. ${ }^{24}$ The RENAL (Radius, Endophytic, Nearness to collecting system, Anterior/posterior and Location) nephrometry scores are also applied in laparoscopic cryoablation, as in laparoscopic partial nephrectomy. The increase in RENAL nephrometry score was noted to be linearly associated with the development of post procedure complications in patients undergoing laparoscopic cryotherapy. This tool is useful in risk stratification, but further work is in progress. ${ }^{25}$

\section{Follow-up after cryoablation}

There is no single accepted protocol for follow up after ablative therapy for SRMs. CT scanning after the administration of intravenous contrast is considered the standard imaging modality. Cryoablation of renal tumour is considered to be successful, if it does not enhance on imaging and shows regression in its size. ${ }^{11}$ The ablated patients in our centre are followed up using a contrast CT scan at 3 and 6 months after the procedure, followed by six monthly imaging, thereafter. Other centres may use slightly differing protocols. Long- term follow is very important to ensure that therapy is successful, as evidenced by regression of tumour size and absence of local recurrence or distant metastases. Contrast enhancement of the ablated tumour or increase in its size, are indicative of local recurrence. However, rim enhancement in a previously cryoablated tumour, in the absence of increase in size, does not always mean the presence of local recurrence. ${ }^{23}$ If there is no satisfactory regression or if local recurrence is suspected, image guided biopsy of the ablated tumour is indicated. ${ }^{26}$

\section{Oncological outcome after Cryoablation}

The oncological success for ablative therapy is promising with high efficiency and low morbidity as seen in both medium and long - term data. $93 \%$ and $81 \%$ cancer survival rates were reported at 5 and 10 years respectively during follow-up. ${ }^{27} \mathrm{~A}$ recent publication by Johnson et al. ${ }^{28}$ reported 112 (T1) SRMs treated with laparoscopic cryoablation and followed up for more than 5 years. At a mean follow up of 98 months, the cancer specific survival was $98.5 \%$, the progression free survival was $91 \%$ and the overall survival was $78 \%$. The relatively lower overall survival rate was attributed to the associated comorbidities of these patients. According to a metaanalysis, the treatment failure rate for cryoablation ranged from $4.6 \%$ to $5.2 \%$ and the metastatic progression rate ranged from $1 \%$ to $1.2 \% .^{29}$ The EuRECA group retrospectively reviewed 808 patients treated with Laparoscopic Cryoablation (LCA) for T1a SRMs from 2005 to 2015 at eight European institutions. Complications were analyzed according to the Clavien-Dindo classification. Kaplan-Meier analyses were used to estimate 5- and 10-year disease-free survival (DFS) and overall survival (OS).The median [interquartile (IQR)] age was 67 (58-74) years. The median (IQR) tumour size was 25 (19-30) $\mathrm{mm}$. The transperitoneal approach was used in $77.7 \%$ of the patients. The median postoperative hospital stay was 2 days. In all, 514 patients with a biopsy-confirmed renal cell carcinoma (RCC) were available for survival analyses. The median (IQR) follow-up for the RCC-cohort was 36 (14-56) months. A total of 32 patients (6.2\%) were diagnosed with treatment failure. The 5 and10-year DFS rate was $90.4 \%$ and $80.0 \%$ respectively. The 5 and 10 -year OS rate was $83.2 \%$ and $64.4 \%$, respectively. A total of 134 postoperative complications (16.6\%) were reported, with severe complications (grade $\geq$ III) in 26 patients (3.2\%). An American Society of Anesthesiologists (ASA) score of 3 was associated with an increased risk of overall complications (odds ratio 2.85, 95\% confidence interval 1.32-6.20; $\mathrm{P}=0.005$ ). ${ }^{24}$ Zargar et al. ${ }^{10}$ recently performed a systematic review of cryotherapy of small renal masses and concluded thatcryoablation had excellent functional outcome and low rate of complications. However, they recommended that patient selection criteria and oncological outcomes needed further evaluation. EuRECA is prospectively collecting data from across Europe and will be able to shed more light in the coming years.

\section{Radio frequency ablation (RFA)}

Radiofrequency ablative therapy was initially used in the treatment of liver lesions. It was first used in kidneys in 1997.30 Although, it can be used laparoscopically, it is mostly applied percutaneously. ${ }^{31}$ Electrodes are inserted into the tumour and high-frequency electrical current is passed creating heat, which destroys the cancer cells, in this technique. The tumour tissue is ablated along with a small margin of normal renal tissue around it, by immediate direct cellular damage and delayed microvascular injury leading to permanent denaturation of the tissue protein. For adequate tissue destruction, the tissue temperature achieved has to be between $50^{\circ} \mathrm{C}$ and $100^{\circ} \mathrm{C}$. There will only be patchy tissue damage, if the temperature achieved rises to $105^{\circ} \mathrm{C}$ or above. As RFA can be performed percutaneously under sedation avoiding the morbidity associated with general anaesthesia, laparoscopy and surgery, it is considered to be advantageous over these procedures. However the main disadvantage with RFA is the unreliability of real time imaging using CT, MRI or US, ${ }^{12}$ due to the similarity between ablated tumour and normal tissue. However, during subsequent follow-up, successfully ablated tumours show regression in size and lack of enhancement with contrast imaging, similar to cryoablation.

\section{Oncological outcome of RFA}

Similar to cryotherapy, RFA also lacks long- term data of their outcome. In a study that followed up SRMs in single kidneys treated with RFA for over 40 months, ${ }^{32}$ the recurrence free survival rate was $90.3 \%$. Disease specific survival rate following RFA in T1a lesions was reported as $93.4 \%$ by Stern et $a .^{33}$ in their study with a mean follow up of 30 months. This outcome is similar to that for tumours of the same stage treated with partial nephrectomy. Tracey et al. ${ }^{34}$ observed in a large study of 243 procedures with a mean follow up of 27 months, a $90 \%$ five year recurrence free survival in histologically proven renal cell cancer. The long- term oncological outcome of SRMs treated with RFA were analysed by Ramirez et al. ${ }^{31}$ The estimated 5 
year recurrence free survival was $93.3 \%$, in 79 patients who had 111 renal masses treated over a 10 year period with a median follow up of 59 months. They had a complication rate of $8.8 \%$ of which $3.8 \%$ were considered major. The long-term oncological outcomes for patients, with $\mathrm{T} 1$ renal cell cancers treated by RFA, were also studied by Psutka et al..$^{35}$ They had 185 patients, of whom 143 had T1atumours and 42 patients had T1b tumours. In multivariate analysis, tumour stage was the only significant predictor of disease free survival. The disease free survival rate was $93 \%$ for $\mathrm{T} 1 \mathrm{a}$ tumours and $76 \%$ for $\mathrm{T} 1 \mathrm{~b}$ tumours, at a median follow up of 6.4 years. Local recurrence was seen in $6(4.2 \%)$ patients in the T1a group and also in $6(14.3 \%)$ patients in the T1b group, with a median time to recurrence of 2.5 years.

\section{Preservation of renal function after ablative treatment}

One of the main advantages of the ablation techniques is that the renal function is not compromised. Woldu et al. ${ }^{36}$ in their recently published retrospective analysis concluded that ablative therapy modalities including cryoablation and RFA were associated with less Renal Parenchymal Volume (RPV) loss compared to partial nephrectomy with its three modalities (open, laparoscopic and robot assisted). Also in patients with chronic kidney disease who had cryoablation, their renal function was maintained. ${ }^{37,38}$ Even in patients with single kidney who had cryotherapy by laparoscopic technique ${ }^{39}$ or by imaging guided percutaneous treatment, ${ }^{32}$ there appears to be no detrimental loss of renal function. The systematic review by Zarga et al. ${ }^{10}$ also reiterated this fact.

\section{Is cryotherapy or RFA, better?}

It is always a debate as to which of these two well established ablative treatments is better? RFA is performed under local anaesthesia with image guidance and is suitable for patients who have significant comorbidity. Although percutaneous cryotherapy is evolving, most groups still tend to do them under general anaesthesia. Cryotherapy demonstrates observable treatment changes during the procedure, which is spectacular under laparoscopy, while real time monitoring of RFA treatment with any of the imaging modality is unreliable. Certainly, the oncological outcomes seem to favour cryotherapy over RFA. The medium and long- term data term data available show that cryoablation may have better tumour control and so less risk of local recurrence. In a meta-analysis, Kunkle et al. ${ }^{20}$ showed that among patients who had RFA and cryoablation for small renal tumours, pretreatment biopsy was done more frequently in tumours treated with cryotherapy $(82.3 \%)$ than those treated with RFA (62.2\%) $(\mathrm{P}<0.0001)$.

Significantly higher number of tumours treated with RFA needed repeat ablations compared to cryotherapy $(8.5 \%$ and $1.3 \%$ respectively) $(p<0.0001)$, in that study. Moreover, the rate of local tumour progression was significantly higher for RFA (12.9\%) than for cryoablation $(5.2 \%) \quad(\mathrm{p}<0.0001)$.There was more metastatic progression $(2.5 \%)$ in the RFA treated group than in the Cryoablation group. The same authors, in another meta-analysis, compared the outcome of nephron sparing surgery, cryoablation and RFA in 99 studies that included 6471 renal masses. The SRMs treated with RFA showed a local recurrence rate of $11.7 \%$ compared to $4.6 \%$ and $2.6 \%$ for the masses treated with cryoablation and nephron sparing surgery respectively. ${ }^{29}$ A recently published retrospective study by Thompson et al..$^{40}$ included $1803 \mathrm{cT} 1$ renal masses treated in Mayo clinic over a period of 11 years, of which, 1057 tumours were treated with laparoscopic partial nephrectomy (LPN), 187 were treated with cryotherapy and 180 with radio frequency ablation (RFA). The recurrence free survival rate was similar in all the three treatment groups, in this study. The overall survival rate was superior in the LPN group and metastasis free survival was significantly lower in the RFA group. However, there was a selection bias with younger patients being offered LPN and so it was considered to be an unmatched retrospective analysis. ${ }^{41}$

\section{High Intensity focused ultrasound (Hifu) ablation}

Focused High Intensity Ultrasonic waves produce the thermal effect required to induce coagulative necrosis in the target tissues, with this modality of treatment. In addition, it also has a direct mechanical destructive effect caused by bubble implosion. The HIFU ablation technique is completely non-invasive, but has disadvantages that include difficulty in localization of the lesions due to overlying rib and respiratory movement and has complications including bowel injury and skin burns. ${ }^{42}$

\section{Conclusion}

With recurrence free survival rates approaching that of extirpative surgery, ablative therapies have emerged as viable treatment options for SRMs. There are minimal and minor complications associated with these procedures. Partial Nephrectomy (PN) remains the gold standard for treatment of SRMs. However, ablation therapy could be a particularly useful alternative in patients whose comorbidity precludes them from safely having a PN or when preservation of renal function is critical, as in patients with single kidneys. The oncological outcomes of ablative therapy are promising as evidenced by short and medium term follow-up data. The efficacy of ablative therapy is improving with the advent of new technologies to aid accurate probe insertion, real time monitoring and energy delivery. However, there is currently no consensus in the literature on the best selection criteria for ablative therapy for SRMs, which needs further refinement. Prospective long- term studies to evaluate oncological outcome are also needed. Newer ablation therapy modalities such as Cyber Knife, Interstitial LASER coagulation and Microwave thermotherapy are still in their experimental stage and lack sufficient data to support their routine use in the current management of SRMs.

\section{Acknowledgement}

None.

\section{Conflicts of interest}

The author declares there is no conflict of interest.

\section{References}

1. Chow WH, Devesa SS, Warren JL, et al. Rising incidence of renal cell cancer in the United States. JAMA. 1999;281(17):1628-1631.

2. Crispen PL, Uzzo RG. The natural history of untreated renal masses. BJU international. 2007;99(5b):1203-1207.

3. Chawla SN, Crispen PL, Hanlon AL, et al. The natural history of observed enhancing renal masses: meta-analysis and review of the world literature. J Urol. 2006;175(2):425-431.

4. Lee CT, Katz J, Shi W, et al. Surgical management of renal tumors $4 \mathrm{~cm}$. or less in a contemporary cohort. J Urol. 2000;163(3):730-736.

5. Lau WK, Blute ML, Weaver AL, et al. Matched comparison of radical nephrectomy vs nephron-sparing surgery in patients with unilateral renal cell carcinoma and a normal contralateral kidney. Mayo Clin Proc. 2000;75(12):1236-1242. 
6. McKiernan J, Simmons R, Katz J, et al. Natural history of chronic renal insufficiency after partial and radical nephrectomy. Urology. 2002;59(6):816-820.

7. Gill IS, Kavoussi LR, Lane BR, et al. Comparison of 1,800 laparoscopic and open partial nephrectomies for single renal tumors. J Urol. 2007;178(1): 41-46.

8. Hadjipavlou M, Khan F, Fowler S, et al. Partial vs radical nephrectomy for T1 renal tumours: an analysis from the British Association of Urological Surgeons Nephrectomy Audit. BJU Int. 2016;117(1):62-71.

9. Gill IS. Minimally invasive nephron-sparing surgery. Urol Clin North Am. 2003;30(3):551-579.

10. Zargar H, Atwell TD, Cadeddu JA, et al. Cryoablation for small rena masses: selection criteria, complications, and functional and oncologic results. Eur Urol. 2016;69(1):116-128.

11. Khan F, Sriprasad S, Keeley FX. Cryosurgical ablation for small rena masses, current status and future prospects. British Journal of Medical and Surgical Urology. 2012;5(1):S28-S34.

12. Sriprasad S, Marsh H. The Management of Small Renal Tumours by Ablative Therapies. INTECH Open Access Publisher. 2011.

13. Rewcastle JC, Sandison GA, Saliken JC, et al. Considerations during clinical operation of two commercially available cryomachines. J Surg Oncol. 1999;71(2):106-111.

14. Acker JP, Larese A, Yang H, et al. Intracellular ice formation is affected by cell interactions. Cryobiology. 1999;38(4):363-371.

15. Bischof JC, Smith D, Pazhayannur PV, et al. Cryosurgery of dunning AT-1 rat prostate tumor: thermal, biophysical, and viability response at the cellular and tissue level. Cryobiology. 1997;34(1):42-69.

16. Daum PS, Bowers WD, Tejada J, et al. Vascular casts demonstrate microcirculatory insufficiency in acute frostbite. Cryobiology. 1987;24(1):65-73

17. Clarke DM, Robilotto AT, Rhee E, et al. Cryoablation of renal cancer: variables involved in freezing-induced cell death. Technol Cancer Res Treat. 2007;6(2):69-79.

18. Janzen N, Zisman A, Pantuck AJ, et al. Minimally invasive ablative approaches in the treatment of renal cell carcinoma. Curr Urol Rep. 2002;3(1):13-20.

19. Chodez M, Fiard G, Arnoux V, et al. Ablative treatments in localised renal cancer: Literature review in 2014. Prog Urol. 2015;25(9):499-509.

20. Kunkle DA, Uzzo RG. Cryoablation or radiofrequency ablation of the small renal mass: a meta-analysis. Cancer. 2008;113(10):2671-2680.

21. Autorino R, Haber GP, White MA, et al. New developments in renal focal therapy. Journal of endourology / J Endourol. 2010;24(5):665-672.

22. Laguna MP, Beemster P, Kumar V, et al. Perioperative morbidity of laparoscopic cryoablation of small renal masses with ultrathin probes: a European multicentre experience. Eur Urol. 2009;56(2):355-361.

23. Lokken RP, Gervais DA, Arellano RS, et al. Inflammatory nodules mimic applicator track seeding after percutaneous ablation of renal tumors. AJR Am J Roentgenol. 2007;189(4):845-848.

24. Nielsen TK, Lagerveld BW, Keeley F, et al. Oncological outcomes and complication rates after laparoscopic-assisted cryoablation: a European Registry for Renal Cryoablation (EuRECA) multi-institutional study. BJU Int. 2016;10.1111/bju.13615
25. Sisul DM, Liss MA, Palazzi KL, et al. RENAL nephrometry score is associated with complications after renal cryoablation: a multicenter analysis. Urology. 2013;81(4):775-780.

26. Beemster P, Phoa S, Wijkstra H, et al. Follow-up of renal masses after cryosurgery using computed tomography; enhancement patterns and cryolesion size. BJU Int. 2008;101(10):1237-1242.

27. Berger A, Kamoi K, Gill IS, et al. Cryoablation for renal tumors: current status. Curr Opin Urol. 2009;19(2):138-142.

28. Johnson S, Pham KN, See W, et al. Laparoscopic cryoablation for clinical stage T1 renal masses: long-term oncologic outcomes at the Medical College of Wisconsin. Urology. 2014;4(3):613-618.

29. Kunkle DA, Egleston BL, Uzzo RG (2008) Excise, ablate or observe: the small renal mass dilemma--a meta-analysis and review. J Urol 179(4): 1227-1233.

30. Weight CJ, Fergany AF, Gunn PW, et al. The impact of minimally invasive techniques on open partial nephrectomy: a 10-year single institutional experience. J Urol. 2008;180(1):84-88.

31. Ramirez D, Ma YB, Bedir S, et al. Laparoscopic radiofrequency ablation of small renal tumors: long-term oncologic outcomes $J$ Endourol. 2014;28(3):330-334

32. Levinson AW, Su LM, Agarwal D, et al. Long-term oncological and overall outcomes of percutaneous radio frequency ablation in high risk surgical patients with a solitary small renal mass. J Urol. 2008;180(2):499504 .

33. Stern JM, Svatek R, Park S, et al. Intermediate comparison of partial nephrectomy and radiofrequency ablation for clinical T1a renal tumours. BJU Int. 2007;100(2):287-290.

34. Tracy CR, Raman JD, Donnally C, et al. Durable oncologic outcomes after radiofrequency ablation. Cancer. 2010;116(13):3135-3142.

35. Psutka SP, Feldman AS, McDougal WS, et al. Long-term oncologic outcomes after radiofrequency ablation for T1 renal cell carcinoma. Eur Urol. 2013;63(3):486-492.

36. Woldu SL, Thoreson GR, Okhunov Z, et al. Comparison of Renal Parenchymal Volume Preservation Between Partial Nephrectomy, Cryoablation, and Radiofrequency Ablation Using 3D Volume Measurements. J Endourol. 2015;29(8):948-955.

37. Michaels MJ, Rhee HK, Mourtzinos AP, et al. Incomplete renal tumor destruction using radio frequency interstitial ablation. J Urol. 2002;168(6):2406-2409.

38. Altunrende F, Autorino R, Hillyer S, et al. Image guided percutaneous probe ablation for renal tumors in 65 solitary kidneys: functional and oncological outcomes. J Urol. 2011;186(1):35-41.

39. Khan F, Mahmalji W, Madaan S. Multi-institutional analysis of renal function following cryoablation of small renal masses in solitary kidneys. In BJU International. 2013;111:37-37.

40. Thompson RH, Atwell T, Schmit G, et al. Comparison of partial nephrectomy and percutaneous ablation for cT1 renal masses. Eur Urol. 2015;67(2):252-259.

41. Kutikov A, Smaldone MC, Uzzo RG. Focal therapy for treatment of the small renal mass: dealer's choice or a therapeutic gamble? Eur Urol. 2015;67(2):260-261.

42. Marberger M, Schatzl G, Cranston D, et al. Extracorporeal ablation of renal tumours with high-intensity focused ultrasound. BJU Int. 2005;95(Suppl 2):52-55. 